\title{
Impact of Gabapentin and Pregabalin on Neurological Outcome After Ischemic Stroke
}

\author{
Michael Weber ${ }^{1}$, Caleb Morton ${ }^{1}$, Fen-Lei Chang ${ }^{1,2}$ \\ ${ }^{1}$ Indiana University School of Medicine-Fort Wayne; ${ }^{2}$ Parkview Health Neurosciences
}

\section{Background}

The purpose of this study is to determine whether patients taking either gabapentin or pregabalin at the time of their stroke injury tend to have better outcomes than patients with similar injuries who were not taking one of the two medications. Prior studies have shown a potential neuroprotective effects of these two medications.

\section{Methods}

A retrospective chart review of 115 ischemic stroke patients from 2016-2021 were assessed for patient outcomes using two tools, the NIH Stroke Scale (NIHSS) and the modified Rankin Scale (mRS), in addition to their hospital length of stay. The outcomes of patients taking either gabapentin or pregabalin with stroke diagnoses are compared to patients with stroke diagnoses who were not taking either medication. Kruskal-Wallace and $\mathrm{X}^{2}$ were used for statistical analysis.

\section{$\underline{\text { Results }}$}

There was significantly larger proportion of gabapentin patients that improved compared to patients in the control group when using the mRS tool for patient outcomes $\left(X^{2} ; p=0.015\right)$. The gabapentin group showed a significantly larger improvement in the NIHSS scores from admission to discharge (Kruskal-Wallace; $p=0.0005$ ). Patients on gabapentin had a longer hospital stay than those not taking the medication by 1.7 days (t-test; $p=0.041$ ).

\section{$\underline{\text { Conclusion }}$}

Our data support the potential neuro-protective effect of gabapentin/pregabalin with improved outcomes after an ischemic stroke using two parallel outcome measures of NIHSS and mRS scores. Of interest, patient hospital stays were longer on gabapentin/pregabalin, which may contribute to the improved outcomes. We need larger subject groups to confirm and further study our findings. This often can be facilitated by studies involving larger medical practices, insurance, or payer databases. In addition, impact of associated cost and care quality issues such as nosocomial infection and fall risk can be considered in the context of healthcare integration and value-based care emphasizing quality and cost management. 